\title{
HARISH-CHANDRA MODULES OVER THE TWISTED HEISENBERG-VIRASORO ALGEBRA
}

\author{
Dong $\mathrm{Liu}^{a}$ and Cuipo Jiang ${ }^{b}$ \\ ${ }^{a}$ Department of Mathematics, Huzhou Teachers College \\ Zhejiang Huzhou, 313000, P.R.China \\ ${ }^{b}$ Department of Mathematics, Shanghai Jiaotong University \\ Shanghai, 200240, P.R.China
}

\begin{abstract}
In this paper, we classify all indecomposable Harish-Chandra modules of the intermediate series over the twisted Heisenberg-Virasoro algebra. Meanwhile, some bosonic modules are also studied.

Key Words: Bosonic modules, Harish-Chandra modules, the twisted Heisneberg-Virasoro algebras.
\end{abstract}

\section{Introduction}

The twisted Heisenberg-Virasoro algebra has been first studied by Arbarello et al. in Ref.[1], where a connection is established between the second cohomology of certain moduli spaces of curves and the second cohomology of the Lie algebra of differential operators of order at most one:

$$
L_{H V}=\left\{f(t) \frac{d}{d t}+g(t) \mid f, g \in \mathbb{C}\left[t, t^{-1}\right]\right\} .
$$

Moreover, the twisted Heisenberg-Virasoro algebra has some relations with the toroidal Lie algebras (see Ref.[6]) and the $N=2$ Neveu-Schwarz superalgebra, which is one of the most important algebraic objects realized in superstring theory (see Ref.[12]).

By definition, as a vector space over $\mathbb{C}$, the twisted Heisenberg-Virasoro algebra $\mathcal{L}$ has a basis $\left\{L(m), I(m), C_{L}, C_{I}, C_{L I}, m \in \mathbb{Z}\right\}$, subject to the following relations:

$$
\begin{aligned}
& {[L(m), L(n)]=(n-m) L(m+n)+\delta_{m+n, 0} \frac{1}{12}\left(m^{3}-m\right) C_{L} ;} \\
& {[I(m), I(n)]=n \delta_{m+n, 0} C_{I} ;} \\
& {[L(m), I(n)]=n I(m+n)+\delta_{m+n, 0}\left(m^{2}-m\right) C_{L I} ;} \\
& {\left[\mathcal{L}, C_{L}\right]=\left[\mathcal{L}, C_{I}\right]=\left[\mathcal{L}, C_{L I}\right]=0 .}
\end{aligned}
$$

Clearly the Heisenberg algebra $H=\mathbb{C}\left\{I(m), C_{I} \mid m \in \mathbb{Z}\right\}$ and the Virasoro algebra Vir = $\mathbb{C}\left\{L(m), C_{L} \mid m \in \mathbb{Z}\right\}$ are subalgebras of $\mathcal{L}$. Moreover, $\mathcal{L}=\oplus_{m \in \mathbb{Z}} \mathcal{L}_{m}$, where $\mathcal{L}_{m}=\mathbb{C}\left\{t^{m} \partial, t^{m}\right\} \oplus$ $\delta_{m, 0} \mathbb{C}\left\{C_{I}, C_{L}, C_{L I}\right\}$, is a $\mathbb{Z}$-graded Lie algebra.

E-mail: liudong@hutc.zj.cn, cpjiang@sjtu.edu.cn 
Arbarello et al. Ref.[1] also proved that when the central element of the Heisenberg subalgebra acts in a non-zero way, an irreducible highest weight $\mathcal{L}$-module is isomorphic to the tensor product of an irreducible $V i r$-module and an irreducible $H$-module. The structure of the irreducible representations for $\mathcal{L}$ at level zero was studied in Ref.[2]. The irreducible weight modules were first studied in Ref.[3], and then classified in Ref.[16]. Some relations between representation theory of the twisted Heisenberg-Virasoro algebra and that of toroidal Lie algebras was studied in Ref.[6], Ref.[7], and Ref.[10].

By definition, a Harish-Chandra module over the twisted Heisenberg-Virasoro algebra $\mathcal{L}$ is an indecomposable weight module $V=\oplus V^{\lambda}$, where $V^{\lambda}=\{v \in V \mid L(0) v=(\lambda+a) v\}$ (for some $a \in \mathbb{C}$ ) and each $V^{\lambda}$ is finite-dimensional. Moreover if each $V_{\lambda}$ is at most one-dimensional, the nontrivial Harish-Chandra module $V$ is called the intermediate series. By $[L(0), L(n)]=n L(n)$ and $[L(0), I(n)]=n I(n)$ for all $n \in \mathbb{Z}^{*}$, we see that if $V^{\lambda} \neq 0$, then $V=\oplus_{n \in \mathbb{Z}} V^{\lambda+n}$ and $\mathcal{L}_{m} V^{\lambda+n} \subset V^{\lambda+n+m}$. Hence $V$ has a structure of $\mathbb{Z}$-graded module.

Some indecomposable modules of the intermediate series over the Lie algebra of differential operators of order at most one, were constructed in Ref.[3]. This construction is based on one kind of modules of the intermediate series over the Virasoro algebra and also needs a strong condition $L( \pm 1) v_{k} \neq 0$ for all $k \in \mathbb{Z}$, which are not including the other two important kinds of Vir-modules. In this paper we obtain all Harish-Chandra modules of the intermediate series over $\mathcal{L}$ based on all three kinds of the Vir-modules of the intermediate series. We obtain seven kinds of new indecomposable modules over the twisted Heisenberg-Virasoro algebra. Especially, we construct one kind of new indecomposable modules from one kind of decomposable modules of the Virasoro algebra.

The above researches may be very helpful in the following researches. It can be used to classify Harish-Chandra modules over the $N=2$ Neveu-Schwarz superalgebra, whose even part is just the twisted Heisenberg-Virasoro algebra (some central elements are not consider here, see Ref.[8]). Moreover, It also may be helpful in the classification of the grade left-symmetric algebra structure over this algebra (see Ref.[11] in the Virasoro case).

Spinor representations for the affine Lie algebras were first developed by Frenkel Ref.[4] and Kac-Peterson Ref.[13] independently. The idea is to use a Clifford algebra with infinitely many generators to construct certain quadratic elements, which, together with the identity element, span an orthogonal affine Lie algebra. Thereafter, Feingold-Frenkel Ref.[5] constructed the socalled fermionic or bosonic representations for all classical affine Lie algebras by using Clifford or Weyl algebras with infinitely many generators. Bosonic representations for the Lie algebra of differential operators were studied in many papers (Ref.[14], [18], [9], etc.). In this paper we also construct the bosonic modules based on such construction over the twisted Heisenberg-Virasoro algebra.

This paper is organized as follows. In Section 2, we construct a bosonic representation for the twisted Heisenberg-Virasoro algebra by the construction over the Lie algebra of differential operators. In Section 3, we classify all Harish-Chandra modules over the twisted HeisenbergVirasoro algebra. Throughout this paper, $\mathbb{Z}, \mathbb{Z}^{*}$ and $\mathbb{C}$ denote the sets of integers, non-zero integers and complex numbers, respectively. 


\section{Bosonic modules over the twisted Heisenberg-Virasoro al- gebra}

Define $\mathcal{S}$ to be the unital associative algebra with infinitely many generators: $a(n), a^{*}(n)(n \in \mathbb{Z})$ with relations

$$
\begin{gathered}
{[a(n), a(m)]=\left[a^{*}(n), a^{*}(m)\right]=0,} \\
{\left[a(n), a^{*}(m)\right]=-\delta_{n+m, 0} .}
\end{gathered}
$$

We define the normal ordering as follows.

$$
: a(n) a^{*}(m):= \begin{cases}a(n) a^{*}(m), & n \leq m, \\ a^{*}(m) a(n), & n>m,\end{cases}
$$

for $n, m \in \mathbb{Z}$. Set

$$
\theta(n)= \begin{cases}1, & n>0 \\ 0, & n \leq 0\end{cases}
$$

Then

$$
a(n) a^{*}(m)=: a(n) a^{*}(m):-\delta_{n+m, 0} \theta(n-m),
$$

and

$$
\begin{gathered}
{\left[a(m) a^{*}(n), a(p)\right]=\delta_{n+p, 0} a(m),} \\
{\left[a(m) a^{*}(n), a^{*}(p)\right]=-\delta_{m+p, 0} a^{*}(n),}
\end{gathered}
$$

for $m, n, p \in \mathbb{Z}$.

Let $\mathcal{S}^{+}$be the subalgebra generated by $a(n), a^{*}(0), a^{*}(m)$ for $n, m>0$. Let $\mathcal{S}^{-}$be the subalgebra generated by $a(0), a(n), a^{*}(m)$ for $n, m<0$. Those generators in $\mathcal{S}^{+}$are called annihilation operators while those in $\mathcal{S}^{-}$are called creation operators. Let $V$ be a simple $\mathcal{S}$-module containing an element $v_{0}$, called a "vacuum vector", and satisfying

$$
\mathcal{S}^{+} v_{0}=0
$$

So all annihilation operators kill $v_{0}$ and

$$
V=\mathcal{S}^{-} v_{0}
$$

Now we may construct a class of bosons on $V$. For any $m \in \mathbb{Z}, n \in \mathbb{N}$, set

$$
f(m, n)=\sum_{i \in \mathbb{Z}}(-i)^{n}: a(m-i) a^{*}(i): .
$$

Although $f(m, n)$ are infinite sums, they are well-defined as operators on $V$. Indeed, for any vector $v \in V=\mathcal{S}^{-} v_{0}$, only finitely many terms in (2.9) can make a non-zero contribution to $f(m, n) v$.

Proposition 2.1. (Ref.[18], [9]) For $m_{1}, m_{2} \in \mathbb{Z}, n_{1}, n_{2} \in \mathbb{N}$, we have

$$
\begin{aligned}
{\left[f\left(m_{1}, n_{1}\right), f\left(m_{2}, n_{2}\right)\right]=} & \sum_{i=0}^{n_{1}}\left(\begin{array}{c}
n_{1} \\
i
\end{array}\right) m_{2}^{i} f\left(m_{1}+m_{2}, n_{1}+n_{2}-i\right) \\
& -\sum_{j=0}^{n_{2}}\left(\begin{array}{c}
n_{2} \\
j
\end{array}\right) m_{1}^{j} f\left(m_{1}+m_{2}, n_{1}+n_{2}-j\right) \\
& +\varphi\left(f\left(m_{1}, n_{1}\right), f\left(m_{2}, n_{2}\right)\right)
\end{aligned}
$$


where $\varphi$ is given by

$$
\varphi\left(f\left(m_{1}, n_{1}\right), f\left(m_{2}, n_{2}\right)\right)=\left\{\begin{array}{l}
0, \text { if } m_{1}=0, \\
(-1)^{n_{1}+1} \delta_{m_{1}+m_{2}, 0} \sum_{i=1}^{m_{1}}\left(m_{1}-i\right)^{n_{1}} i^{n_{2}}, \text { if } m_{1}>0, \\
(-1)^{n_{1}} \delta_{m_{1}+m_{2}, 0} \sum_{i=m_{1}}^{-1}\left(m_{1}-i\right)^{n_{1}} i^{n_{2}}, \text { if } m_{1}<0 .
\end{array}\right.
$$

Let $T=f(0,0)$, then

$$
[T, a(n)]=a(n), \quad\left[T, a^{*}(n)\right]=-a^{*}(n),
$$

for all $n \in \mathbb{Z}$. For any $v=a\left(n_{1}\right) \cdots a\left(n_{s}\right) a^{*}\left(m_{1}\right) \cdots a^{*}\left(m_{l}\right) v_{0} \in V$, noting that $T v_{0}=0$, one has

$$
T v=(s-l) v .
$$

According to Proposition 2.1, we obtain

Theorem 2.2. $V$ is a module for the Lie algebra $\mathcal{L}$ with central charge $C_{I}=2, C_{L I}=1 / 2, C_{L}=1$ under the action given by

$$
\begin{gathered}
\pi(L(m))=f(m, 1), \quad \pi(I(m))=f(m, 0), \\
\pi\left(C_{L}\right)=2 i d, \quad \pi\left(C_{L I}\right)=\frac{1}{2} i d, \quad \pi\left(C_{I}\right)=1 i d,
\end{gathered}
$$

for all $m \in \mathbb{Z}$. Moreover,

$$
V=\bigoplus_{k \in \mathbb{Z}} V_{k}
$$

is completely reducible, where $V_{k}$ is an eigenspace with eigenvalue $k$ of operator $T$, and each component $V_{k}$ is irreducible as a $\mathcal{L}$-module.

Proof. Note that

$$
\varphi(f(m, 1), f(n, 1))=\left\{\begin{array}{l}
0, \text { if } m=0 \\
\delta_{m+n, 0} \sum_{i=1}^{m}(m-i) i=\delta_{m+n, 0} \frac{m^{3}-m}{6}, \text { if } \quad m>0 \\
-\delta_{m+n, 0} \sum_{i=m}^{-1}(m-i) i=\delta_{m+n, 0} \frac{m^{3}-m}{6}, \text { if } \quad m<0
\end{array} .\right.
$$

So

$$
\varphi(f(m, 1), f(n, 1))=\delta_{m+n, 0} \frac{m^{3}-m}{6} .
$$

Similarly,

$$
\begin{gathered}
\varphi(f(m, 1), f(n, 0))=\delta_{m+n, 0} \frac{m(m-1)}{2} . \\
\varphi(f(m, 0), f(n, 0))=n \delta_{m+n, 0} .
\end{gathered}
$$

Hence, Proposition 2.1 shows that $V$ is a $\mathcal{L}$-module with central charge $C_{L}=2, C_{L I}=$ $1 / 2, C_{I}=1$.

The second statement is essentially same as the proof of Theorem 2.3 in Ref.[9]. 


\section{Harish-Chandra modules of the intermediate series over the twisted Heienberg-Virasoro algebra}

Kaplansky-Santharoubane Ref.[15] in 1983 gave a classification of Vir-modules of the intermediate series. There are three families of indecomposable modules of the intermediate series (i.e nontrivial indecomposable weight modules with each weight space is at most one-dimensional) over the Virasoro algebra. They are Vir-modules "without central charge".

(1) $\mathcal{A}_{a, b}=\sum_{i \in \mathbb{Z}} \mathbb{C} v_{i}: L(m) v_{i}=(a+i+b m) v_{m+i}$;

(2) $\mathcal{A}(a)=\sum_{i \in \mathbb{Z}} \mathbb{C} v_{i}: L(m) v_{i}=(i+m) v_{m+i}$ if $i \neq 0, L(m) v_{0}=m(m+a) v_{m}$;

(3) $\mathcal{B}(a)=\sum_{i \in \mathbb{Z}} \mathbb{C} v_{i}: L(m) v_{i}=i v_{m+i}$ if $i \neq-m, L(m) v_{-m}=-m(m+a) v_{0}$, for some $a, b \in \mathbb{C}$.

When $a \notin \mathbb{Z}$ or $b \neq 0,1$, it is well-known that the module $\mathcal{A}_{a, b}$ is simple. In the opposite case the module contains two simple subquotients namely the trivial module and $\mathbb{C}\left[t, t^{-1}\right] / \mathbb{C}$. Denote the nontrivial simple subquotients of $\mathcal{A}_{a, b}, \mathcal{A}(a), \mathcal{B}(a)$ by $\mathcal{A}_{a, b}^{\prime}, \mathcal{A}(a)^{\prime}, \mathcal{B}(a)^{\prime}$ respectively. They are all Harish-Chandra modules of the intermediate series over the Virasoro algebra.

Furthermore, we have

Lemma 3.1. Ref.[15] Among the above modules, we have following module isomorphisms:

(i) $\mathcal{A}_{a, b} \cong \mathcal{A}_{a^{\prime}, b}$ if $a-a^{\prime} \in \mathbb{Z}$,

(ii) $\mathcal{A}_{a, 0} \cong \mathcal{A}_{a^{\prime}, 1}$ for $a \notin \mathbb{Z}$ with $a-a^{\prime} \in \mathbb{Z}$,

(iii) $\mathcal{A}(a)^{\prime} \cong \mathcal{B}(b)^{\prime} \cong \mathcal{A}_{0,0}^{\prime}$ for all $a, b \in \mathbb{C}$.

Theorem 3.2. Ref.[17] Let $V$ be a $\mathbb{Z}$-graded Vir-module with $\operatorname{dim} V>1$ and $\operatorname{dim} V_{j} \leq 1$ for all $j \in \mathbb{Z}$. Suppose there exists $a \in \mathbb{C}$ such that $L_{0}$ acts on $V_{j}$ as the scalar $a+j$. Then $V$ is isomorphic to one of the following for appropriate $a, b$ : (i) $\mathcal{A}_{a, b}^{\prime}$, (ii) $\mathcal{A}_{0,0}^{\prime} \oplus \mathbb{C} v_{0}$ as direct sum of Vir-modules, (iii) $\mathcal{A}(a)$, (iv) $\mathcal{B}(a)$.

For $a, b, c, d \in \mathbb{C}$, we can similarly define some $H_{V i r}$-modules with $C_{L I}=C_{I}=C_{L}=0$ as follows:

(1) $\mathcal{A}_{a, b, c}: L(n) v_{t}=(a+t+b n) v_{n+t}, I(n) v_{t}=c v_{n+t}$;

(2) $\mathcal{A}(a, d): L(n) v_{t}=(t+n) v_{n+t}$ if $t \neq 0, L(n) v_{0}=n(n+a) v_{n}, I(n) v_{t}=0$ if $t \neq 0$, $I(n) v_{0}=d n v_{n}$

(3) $\mathcal{B}(a, d): L(n) v_{t}=t v_{n+t}$ if $t \neq-n, L(n) v_{-n}=-n(n+a) v_{0}, I(n) v_{t}=0$ if $n+t \neq 0$, $I(n) v_{-n}=d n v_{0}$.

(4) $\mathcal{U}_{d}: L(n) v_{t}=t v_{n+t}, I(n) v_{t}=0$ if $n+t \neq 0, I(n) v_{-n}=n d v_{0}$;

$(5) \mathcal{V}_{d}: L(n) v_{t}=(t+n) v_{n+t}, I(n) v_{t}=0$ if $t \neq 0, I(n) v_{0}=n d v_{n}$;

(6) $\tilde{\mathcal{U}}_{d}:=\sum_{m \in M} \mathbb{C} v_{m}: L(n) v_{t}=t v_{n+t}$ if $n+t \neq 0, L(n) v_{-n}=0, I(n) v_{t}=0$ if $n+t \neq 0$, $I(n) v_{-n}=d n v_{0}, d \in \mathbb{C}^{*}$.

(7) $\tilde{\mathcal{V}}_{c}:=\sum_{m \in M} \mathbb{C} v_{m}: L(n) v_{t}=t v_{n+t}$ if $n+t \neq 0, L(n) v_{-n}=0, I(n) v_{t}=0$ if $t \neq 0$ or $n=t=0 ; I(n) v_{0}=c v_{n}, n \neq 0, c \in \mathbb{C}^{*}$.

It is easy to prove that $\mathcal{A}_{a, b, c}$ is simple if and only if $a \notin M$ or $b \notin\{0,1\}$ or $c \neq 0$. For $a \in \mathbb{Z}$, the module $\mathcal{A}_{a, 0,0}$ has an irreducible quotient $\mathcal{A}_{a, 0,0}^{\prime}=\mathcal{A}_{a, 0,0} / \mathbb{C} v_{-a}$ and $\mathcal{A}_{a, 1,0}$ contains an irreducible submodule $\mathcal{A}_{a, 1,0}^{\prime}=\oplus_{n \neq-a} \mathbb{F} v_{n}$. 
Clearly, the above modules are indecomposable and are not isomorphic each other. Now we shall classify all such modules, i.e., all indecomposable weight modules with all weight multiplicities $\leq 1$ over the generalized Heisenberg-Virasoro algebra. First we have the following result.

Theorem 3.3. Among the $\mathcal{L}$-modules $\mathcal{A}_{a, b, c}$ for $a, b, c \in \mathbb{C}$, and their nontrivial subquotients, we have following module isomorphisms:

(i) $\mathcal{A}_{a, b, c} \cong \mathcal{A}_{a^{\prime}, b^{\prime}, c}$ if $a-a^{\prime} \in \mathbb{Z}$,

(ii) $\mathcal{A}_{a, 0, c} \cong \mathcal{A}_{a^{\prime}, 1, c}$ for $a \notin \mathbb{Z}$ with $a-a^{\prime} \in \mathbb{Z}$,

Proof. It is clear.

Now we shall classify all Harish-Chandra modules over the twisted Heisenberg-Virasoro algebra. First we have the following two lemmas.

Lemma 3.4. Let $V=\sum_{t \in \mathbb{Z}} \mathbb{C} v_{t}$ be a $M$-graded $\mathcal{L}$-module such that $L(n) v_{t}=(a+t+b n) v_{t+n}$ for all $n, t \in \mathbb{Z}$.

(i) If $a+b n \neq 0$ for all $n \in \mathbb{Z}$, and $b \neq 0,1$. Then

$$
I(m) v_{t}=c v_{m+t}
$$

for some $c \in \mathbb{C}$.

(ii) If $a \notin \mathbb{Z}$ and $b=0$, then

(1) $I(m) v_{t}=c v_{m+t}$, in this case $V \cong \mathcal{A}_{a, 0, c}$

or

(2) $I(n) v_{t}=\frac{t+a}{n+t+a} c v_{n+t}$ for all $m, t \in M$, in this case $V \cong \mathcal{A}_{a, 1, c}$ by setting $v_{t}^{\prime}=\frac{1}{a+t} v_{t}$.

Proof. (i) Since $V$ is a module of the Virasoro algebra $\operatorname{Vir}=\oplus_{m \in \mathbb{Z}} L(m)$, it is clear that $C_{L}=0$ (cf. [SZ] for example). Suppose that $I(n) v_{t}=f(n, t) v_{n+t}$ for all $n, t \in \mathbb{Z}$. From $[L(n), I(m)]=$ $m I(n+m)+\delta_{n+m, 0}\left(n^{2}-n\right) C_{L I}$, we see that

$$
\begin{gathered}
f(m, t) L(n) v_{t+m}-f(m, n+t)(a+t+b n) v_{n+t+m} \\
=m f(n+m, t) v_{n+m+t}+\delta_{n+m, 0}\left(n^{2}-n\right) C_{L I} v_{t} .
\end{gathered}
$$

In this case,

$$
f(m, t)(a+t+m+b n)-f(m, n+t)(a+t+b n)=m f(n+m, t)+\delta_{n+m, 0}\left(n^{2}-n\right) C_{L I} .
$$

Let $m=0$ in (3.2), then we have

$$
f(0, t)=f(0, n+t) .
$$

Let $t=0$ in (3.2), then

$$
f(m, n)(a+b n)=(a+m+b n) f(m, 0)-\left(m f(n+m, 0)+\delta_{n+m, 0}\left(n^{2}-n\right) C_{L I}\right), \quad n \neq 0 .
$$

Setting $n=-2 m$ in (3.4), we have

$$
(a-2 m b) f(m,-2 m)=(a+m-2 m b) f(m, 0)-m f(-m, 0) .
$$

Let $n=t=-m$ in (3.2), then

$$
f(m,-m)(a-b m)-f(m,-2 m)(a-(1+b) m)=m f(0,-m)+\left(m^{2}+m\right) C_{L I} .
$$


Setting $n=-m$ in (3.4), then

$$
(a-m b) f(m,-m)=(a+m-b m) f(m, 0)-m f(0,0)-\left(m^{2}+m\right) C_{L I} .
$$

From (3.6) and (3.7) we obtain that

$$
-(a-m-b m) f(m,-2 m)=2 m f(0,0)-(a+m-b m) f(m, 0)+2\left(m^{2}+m\right) C_{L I} .
$$

Therefore from (3.5) and (3.8) we have

$$
\begin{aligned}
& (a+(1-3 b) m) f(m, 0)+(a-(1+b) m) f(-m, 0) \\
= & 2(a-2 b m) f(0,0)+2(a-2 b m)(1+m) C_{L I}, \quad m \neq 0 .
\end{aligned}
$$

Replacing $m$ by $-m$ in (3.9), (3.9) becomes

$$
\begin{aligned}
& (a+(1+b) m) f(m, 0)+(a-(1-3 b) m) f(-m, 0) \\
= & 2(a+2 b m) f(0,0)+2(a+2 b m)(1-m) C_{L I}, \quad m \neq 0 .
\end{aligned}
$$

From (3.9) and (3.10), we obtain

$$
2\left(m^{2} b-m^{2} b^{2}\right)(f(m, 0)-f(0,0))=\left(a^{2} m-a m^{2}+2 m^{2} b-2 m^{2} b^{2}-4 m^{3} b^{2}+m^{2} a b\right) C_{L I} .
$$

Setting $t=m=-n$ in (3.2), we have

$$
(a+2 m-b m) f(m, m)=m f(0,0)+(a+m-b m) f(m, 0)+\left(m^{2}+m\right) C_{L I} .
$$

Let $m=n$ in (3.4), then

$$
(a+b m) f(m, m)=(a+m+b m) f(m, 0)-m f(2 m, 0) .
$$

From (3.12) and (3.13) we have

$$
(a+b m)\left(m f(0,0)+\left(m^{2}+m\right) C_{L I}\right)-2\left(m^{2}+a m\right) f(m, 0)+m(a+2 m-b m) f(2 m, 0)=0 .
$$

Applying (3.11) to $f(m, 0)$ and $f(2 m, 0)$, we have

$$
\left(6\left(b^{3}-b^{2}\right) m^{5}+3 a\left(b-b^{2}\right) m^{4}-2 b^{2} m^{4}-2 a b^{2} m^{3}-\frac{3}{2} a^{2} b m^{3}-\frac{3}{2} a^{2} m^{2}\right) C_{L I}=0 .
$$

Since the equation (3.15) has infinitely many solutions and $b(1-b) \neq 0$, considering the coefficient of $m^{5}$, we deduce that

$$
C_{L I}=0
$$

Therefore

$$
f(m, 0)=f(0,0),
$$

for all $m \in \mathbb{Z}$. By (3.4) we obtain that $f(m, n)=f(0,0)=c \in \mathbb{C}$ for all $m, n \in \mathbb{Z}$. It follows that $C_{I}=0$.

(ii) If $a \notin \mathbb{Z}$ and $b=0$, then (3.1)—(3.15) still hold. From (3.11) we know that

$$
\left(a^{2} m-a m^{2}\right) C_{L I}=0, m \neq 0,
$$

i.e.,

$$
a m(a-m) C_{L I}=0, m \neq 0 .
$$


Since $a \notin M$, we have $C_{L I}=0$. Thus (3.2) and (3.4) become

$$
(a+t+m) f(m, t)-(a+t) f(m, n+t)=m f(m+n, t)
$$

and

$$
(a+m) f(m, 0)-a f(m, n)=m f(m+n, 0) .
$$

Setting $n=-m$ in (3.18), we have

$$
(a+m) f(m, 0)-a f(m,-m)=m f(0,0) .
$$

Let $n=m=-t$ in (3.17), then

$$
a f(m,-m)-(a-m) f(m, 0)=m f(2 m,-m) .
$$

By (3.18), we have

$$
a f(2 m,-m)=(a+2 m) f(2 m, 0)-2 m f(m, 0) .
$$

From (3.19) and (3.20), we have

$$
2 m f(m, 0)=m f(2 m,-m)+m f(0,0) .
$$

Therefore

$$
(a+2 m) f(2 m, 0)-2(m+a) f(m, 0)+a f(0,0)=0 .
$$

Furthermore, we have

$$
(k m+a) f(k m, 0)=k(m+a) f(m, 0)-(k-1) a f(0,0) .
$$

By $[I(m), I(n)]=\delta_{m+n, 0} n C_{I}$, we get

$$
f(n, t) f(m, n+t)=f(m, t) f(n, m+t)+\delta_{m+n, 0} n C_{I} .
$$

So

$$
f(2 m, 0) f(m, 2 m)=f(m, 0) f(2 m, m) .
$$

By (3.18) we obtain

$$
\begin{gathered}
f(m, 2 m)=\frac{a+m}{a} f(m, 0)-\frac{m}{a} f(3 m, 0) . \\
f(2 m, m)=\frac{a+2 m}{a} f(2 m, 0)-\frac{2 m}{a} f(3 m, 0) .
\end{gathered}
$$

By (3.24), we have

$$
(3 m+a) f(3 m, 0)=3(m+a) f(m, 0)-2 a f(0,0) .
$$

Combining (3.23) and (3.26) - (3.29) we obtain

$$
f(m, 0)=f(0,0), \quad \text { or } \quad f(m, 0)=\frac{a}{a+m} f(0,0) .
$$

So by (3.18) we have

$$
f(m, n)=f(0,0), \quad \text { or } \quad f(m, n)=\frac{a+n}{a+m+n} f(0,0)
$$

and the lemma is proved.

Remark. 1. In the following cases, we can also deduce that $C_{L}=C_{L I}=C_{I}=0$ as in Lemma 3.4. So in the following discussions, we always assume that $C_{L}=C_{L I}=C_{I}=0$. 
2. In Ref.[3], there is also a similar result (see Proposition 5.1 in Ref.[3]) as Lemma 3.4 on the Lie algebra $L_{H V}$, the Lie algebra of differential operators of order at most one. But it needs a strong condition $L( \pm 1) v_{i} \neq 0$ for all $i \in \mathbb{Z}$ and its proof is more complicated. The two kinds of modules for $b=0$ and $b=1$ in Proposition 5.1 of Ref.[3] are isomorphic if $\alpha \notin \mathbb{Z}$.

Now we prove the main theorem of this section.

Theorem 3.5. Let $V=\sum_{m \in \mathbb{Z}} \mathbb{F} v_{m}$ be an indecomposable $\mathcal{L}$-module such that $\mathcal{L}_{m} v_{n} \in \mathbb{C} v_{m+n}$ for all $m, n \in \mathbb{Z}$. Then $V$ is isomorphic to one of the modules $\mathcal{A}_{a, b, c}, \mathcal{A}(a, c), \mathcal{B}(a, c), \mathcal{U}_{d}, \mathcal{V}_{d}, \tilde{\mathcal{U}}_{d}, \tilde{\mathcal{V}}_{c}$ for appropriate $a, b, c, d \in \mathbb{F}$.

Proof. First we suppose that $V$ is an indecomposable module of the Virasoro algebra Vir= $\oplus_{m \in \mathbb{Z}} L(m)$, then $V$ is isomorphic to one of the modules in Theorem 3.3 as a Vir-module. Suppose that $I(n) v_{t}=f(n, t) v_{n+t}$ for all $t, n \in \mathbb{Z}$.

Case I. $L(n) v_{t}=(a+t+b n) v_{n+t}$ for all $n, t \in \mathbb{Z}$.

(I.1) Suppose that $a \notin \mathbb{Z}$ and $a+b n \neq 0$ for all $n \in \mathbb{Z}$. If $b \neq 0,1$, then $f(m, n)=f(0,0)$ for all $m, n \in \mathbb{Z}$ by Lemma 3.4.

If $b=0$, then $V$ is isomorphic to $\mathcal{A}_{a, 0, c}$ or $\mathcal{A}_{a, 1, c}$ by Lemma 3.4.

If $b=1$, then $V$ is also isomorphic to $\mathcal{A}_{a, 0, c}$ or $\mathcal{A}_{a, 1, c}$ since $\mathcal{A}_{a, 0} \cong \mathcal{A}_{a, 1}$ for $a \notin \mathbb{Z}$.

(I.2) $a \notin \mathbb{Z}$ and $a=b p$ for some $p \in M \backslash\{0\}$. So $b \neq 0,1$. Therefore $f(m, 0)=f(0,0)$ and by $(3.4)$

$$
f(m, n)=f(0,0) \text { if } n+p \neq 0 .
$$

It follows from (3.2) that

$$
f(0,-p)=f(0,0) .
$$

Setting $t=-p, n=-m$ and $m \neq p$ in (3.2) and using (3.31)-(3.32), we have

$$
f(m,-p)(a-p+m-b m)=f(0,0)(a-p+m-b m) .
$$

Since $a=b p$ and $m \neq p$, we have $f(m,-p)=f(0,0)$ if $m \neq p$. Finally, setting $m=n=p, t=-p$ in (3.2) we have $f(p,-p)=f(0,0)$. Therefore $f(m, n)=f(0,0)$ for all $m, n \in \mathbb{Z}$.

(I.3) $a \in \mathbb{Z}$. Since $\mathcal{A}_{a, b} \cong \mathcal{A}_{0, b}$, so we can suppose that $L(n) v_{t}=(t+b n) v_{n+t}$ for all $n, t \in \mathbb{Z}$.

(I.3.1) $b \neq 0,1$, then $a+b n \neq 0$ for all $n \neq 0$. So $f(m, n)=f(0,0)$ by Lemma 3.4. Therefore $V$ is isomorphic to $\mathcal{A}_{0, b, c}$.

(I.3.2) $\quad b=1$. In this case (3.3) still holds and (3.2) and (3.4) becomes

$$
f(m, t)(t+m+n)-f(m, n+t)(t+n)=m f(n+m, t)
$$

and

$$
n f(m, n)=-m f(n+m, 0)+(n+m) f(m, 0)
$$

Replacing $t$ by $n$ and letting $n=-m$ in (3.33) and using (3.3), we have

$$
n f(m, n)=(n-m) f(m, n-m)+m f(0,0) .
$$

Replacing $n$ by $n-m$ in (3.34), we have

$$
(n-m) f(m, n-m)=-m f(n, 0)+n f(m, 0) .
$$


From (3.34)-(3.36), we have

$$
f(n+m, 0)=f(n, 0)+f(m, 0)-f(0,0) .
$$

We deduce that there exists $d \in \mathbb{C}$ such that

$$
f(m, 0)=d m+f(0,0),
$$

for all $m \in \mathbb{Z}$. Applying (3.37) to (3.34) and using (3.38), we have

$$
f(m, n)=f(0,0), n \neq 0 .
$$

Therefore

$$
f(m, n)=\left\{\begin{array}{l}
f(0,0), \quad n \neq 0, \\
d m+f(0,0), \quad n=0, \quad m \neq 0 .
\end{array}\right.
$$

Since $[I(m), I(n)]=m \delta_{m+n, 0} C_{I}$, we have

$$
f(m, n+t) f(n, t)=f(n, m+t) f(m, t) .
$$

Let $t=0, n \neq 0, m \neq 0$ and $m \neq n$, then

$$
f(0,0)(f(0,0)+d n)=f(0,0)(f(0,0)+d m) .
$$

Therefore if $f(0,0) \neq 0$, then $d=0$ and $V \cong \mathcal{A}_{0,1, c}$, where $c=f(0,0) \neq 0$. If $f(0,0)=0$, then

$$
f(m, n)=f(0,0)=0, n \neq 0 .
$$

Therefore

$$
f(m, n)=\left\{\begin{array}{l}
0, \quad n \neq 0 \\
d m, \quad n=0
\end{array}\right.
$$

and $V \cong \mathcal{V}_{d}$.

(I.3.3) $b=0 .(3.2)$ becomes

$$
(t+m) f(m, t)-t f(m, n+t)=m f(m+n, t) .
$$

Let $t=0$ and $n=-m$ in (3.39), then

$$
f(m, 0)=f(0,0)
$$

Setting $t=m, n=-m$ in (3.39) and using (3.40), we have

$$
f(m, m)=0 .
$$

Let $n+t=-m$ in (3.39), then

$$
t f(m,-m)+m f(-t, t)=(t+m) f(m, t) .
$$

Therefore

$$
f(m,-m)+f(-m, m)=2 f(0,0) .
$$

Setting $t=-m-n$ in (3.39), we have

$$
(-n) f(m,-m-n)+(m+n) f(m,-m)=m f(m+n,-m-n) .
$$


Replace $m$ by $n^{\prime}$ and $n$ by $m^{\prime}$ respectively, then

$$
\left(-m^{\prime}\right) f\left(n^{\prime},-m^{\prime}-n^{\prime}\right)+\left(m^{\prime}+n^{\prime}\right) f\left(n^{\prime},-n^{\prime}\right)=n^{\prime} f\left(m^{\prime}+n^{\prime},-m^{\prime}-n^{\prime}\right) .
$$

Let $t=-m$ and replace $n$ by $-n$, then

$$
f(m,-n-m)=f(m-n,-m), m \neq 0 .
$$

Setting $n^{\prime}=m-n, m^{\prime}=n$ in (3.45) and using (3.46), we have

$$
(-n) f(m,-m-n)+m f(m-n,-m+n)=(m-n) f(m,-m) .
$$

From (3.44) and (3.47), we have

$$
2 f(m,-m)-f(m-n,-m+n)=f(m+n,-m-n), m \neq 0 .
$$

Replace $m$ by $n$ and $n$ by $m$ respectively and use (3.43), then

$$
2 f(n,-n)+f(m-n,-m+n)-2 f(0,0)=f(m+n,-m-n) n \neq 0 .
$$

From (3.47) and (3.49), we have

$$
f(m+n,-m-n)-f(0,0)=f(m,-m)-f(0,0)+f(n,-n)-f(0,0) .
$$

We deduce that there exists $d \in \mathbb{C}$ such that

$$
f(m,-m)=d m+f(0,0),
$$

for all $m \in \mathbb{Z}$. By (3.44), we have

$$
f(m, n)=f(0,0)
$$

for all $m, n \in \mathbb{Z}$ and $m+n \neq 0$. So

$$
f(m, n)=\left\{\begin{array}{lc}
d m+f(0,0), & m+n=0, \\
f(0,0), & m+n \neq 0
\end{array} .\right.
$$

As in the above case we can deduce that if $f(0,0) \neq 0$, then $d=0$ and $V \cong \mathcal{A}_{0,0, c}$, where $c=f(0,0) \neq 0$. If $f(0,0)=0$, then

$$
f(m, n)=\left\{\begin{array}{ll}
d m, & m+n=0 \\
0, & m+n \neq 0
\end{array} .\right.
$$

Therefore $V \cong \mathcal{U}_{d}$.

Case II. $L(n) v_{t}=(t+n) v_{n+t}$ if $t \neq 0, L(n) v_{0}=n(n+a) v_{n}$ for some $a \in \mathbb{C}$. We can deduce that $f(m, t)=\left\{\begin{array}{ll}d m, & t=0 \\ 0, & t \neq 0\end{array}\right.$. Therefore $V \cong \mathcal{A}(a, d)$.

Case III. $L(n) v_{t}=t v_{n+t}$ if $t \neq-n, L(n) v_{-n}=-n(n+a) v_{0}$, for some $a \in \mathbb{F}$.

We can deduce that $f(m, t)=\left\{\begin{array}{rr}d m, & t+m=0 \\ 0, & t+m \neq 0\end{array}\right.$. Then $V$ is isomorphic to $\mathcal{B}(a, d)$.

Finally suppose that $V$ is decomposable as a Vir-module. Then $V=\mathcal{A}_{0,0}^{\prime} \oplus \mathbb{C} v_{0}$ by Theorem 3.3. So we can suppose that $L(n) v_{t}=t v_{n+t}$ if $n+t \neq 0, L(n) v_{-n}=0$. Then by calculation as in Case III we can deduce that $I(n) v_{t}=\left\{\begin{array}{ll}d n v_{0} & n+t=0 \\ 0, & n+t \neq 0\end{array}\right.$ or $I(n) v_{t}=\left\{\begin{array}{ll}c v_{n} & n \neq 0, t=0 \\ 0, & \text { otherwises }\end{array}\right.$. 
In fact, in this case (3.2) becomes

$$
\begin{gathered}
(t+m) f(m, t)-t f(m, n+t)=m f(m+n, t), \quad m+n+t \neq 0, n+t \neq 0 . \\
t f(-(n+t), n+t)=(n+t) f(-t, t), \quad n+t \neq 0 . \\
(t+m) f(m, t)=m f(m-t, t), \quad m \neq 0 .
\end{gathered}
$$

If $t \neq 0$ in (3.51), we have

$$
\frac{f(-(n+t), n+t)}{n+t}=\frac{f(-t, t)}{t} .
$$

So

$$
f(m,-m)=d m, \quad \forall m \in \mathbb{Z}^{*}
$$

for some $d \in \mathbb{C}$.

Setting $m=-t$ in (3.52) we get

$$
f(-2 t, t)=0, \quad t \neq 0 .
$$

Setting $t=0$ in (3.51) we have

$$
f(0,0)=0 .
$$

Hence

$$
f(-2 t, t)=0, \quad \forall t \in \mathbb{Z} .
$$

Setting $m=-2 t,-3 t, \cdots$ in (3.52) again, and by induction we get

$$
f(-k t, t)=0, \quad k \neq 1, k \in \mathbb{Z}^{+} .
$$

Setting $t=0$ in (3.50) we get

$$
f(m, 0)=f(n, 0), \quad m \neq 0, n \neq 0 .
$$

Setting $m=-t$ in (3.50) we get

$$
f(-t, n+t)=f(-t+n, t) \quad t \neq 0, n+t \neq 0 .
$$

Setting $n=-k t, k \neq 1, k \in \mathbb{Z}^{+}$in (3.57) we get

$$
f(-t,-(k-1) t)=0,
$$

it is,

$$
f(t, k t)=0, \quad k \in \mathbb{Z}^{+} .
$$

Using (3.55), (3.58), (3.57) and (3.52) repeatedly, we get

$$
f(k t, t)=f(t, k t)=0, \quad \forall k \in \mathbb{Z}, k \neq-1,0 .
$$

Setting $n=t$ in (3.57) and using (3.59), we get

$$
f(0, t)=0, \quad t \neq 0 .
$$


By $[I(m), I(n)]=\delta_{m+n, 0} n C_{I}$, we get

$$
f(n, t) f(m, n+t)=f(m, t) f(n, m+t) .
$$

Setting $m=t$ in (3.61) and using (3.58), we get

$$
f(n, t) f(t, n+t)=0, \quad \forall n, t \in \mathbb{Z} .
$$

Setting $m=t \neq 0$ in (3.50)

$$
f(t, n+t)=f(n+t, t) \quad n+t \neq 0, n+2 t \neq 0, t \neq 0 .
$$

Setting $m=n+t$ in (3.52) we get

$$
f(n+t, t)=\frac{n+t}{n+2 t} f(n, t), \quad n+t \neq 0, n+2 t \neq 0 .
$$

Combining (3.62), (3.63) and (3.64), we get

$$
f(n, t)=0, \quad n+t \neq 0, n+2 t \neq 0, t \neq 0 .
$$

So by (3.59) we have

$$
f(n, t)=0, \quad n+t \neq 0, t \neq 0 .
$$

If $d \neq 0$, then by setting $n=-t \neq 0$ in (3.62), we get

$$
f(t, 0)=0 .
$$

Therefore

$$
f(m, n)=\left\{\begin{array}{lc}
d m & n+m=0 \\
0, & n+t \neq 0
\end{array} .\right.
$$

Therefore $V \cong \tilde{\mathcal{U}}_{d}$.

If $d=0$, then $f(m, 0)=c, m \neq 0$ for some $c \in \mathbb{C}^{*}$

$$
f(m, n)=\left\{\begin{array}{cc}
c & m \neq 0, n=0 \\
0, & \text { otherwises }
\end{array} .\right.
$$

It is easy to see that $f(m, n)$ satisfies

$$
f(m, n+t) f(n, t)=f(n, m+t) f(m, t) .
$$

Therefore $V \cong \tilde{\mathcal{V}}_{c}$ (if $c=0, V$ is decomposable).

The above modules (if they are irreducible) or their nontrivial simple subquotients are the Harish-Chandra modules of the intermediate series. Moreover the above results immediately yield the following theorem.

Theorem 3.6. Any Harish-Chandra module of intermediate series over the Heisenberg-Virasoro algebra is isomorphic to one of above modules (if they are irreducible) or their nontrivial simple subquotients.

Remarks. 1. Irreducible highest weight modules of $\mathcal{L}$ were classified in Ref.[1] and Ref.[2]. Irreducible Harish-Chandra modules of $\mathcal{L}$ were classified in Ref.[16] 
Theorem 3.7. Ref.[16] Any irreducible Harish-Chandra module over the twisted HeisenbergVirasoro algebra is a highest weight module, a lowest weight module or a module of the intermediate series.

2. The above results can be used to classify Harish-Chandra modules of the $N=2$ NeveuSchwarz superalgebra, whose even part is just the twisted Heisenberg-Virasoro algebra except for some central elements. Especially, all Harish-Chandra modules of the intermediate series over this superalgebra from Theorem 3.6 can be constructed (see Ref.[8]).

\section{ACKNOWLEDGMENTS}

The part of this work was done during the visit of the first author in the Department of Mathematics at University of Bielefeld. He would like to expresses his special gratitude to the

'AsiaLink Project' for financial support. He is also deeply indebted to Prof. C.M. Ringel for his kind hospitality and continuous encouragement and instruction. The project is supported by the NNSF (Grant 10671027, 10701019, 10571119), the ZJZSF(Grant Y607136), and Qianjiang Excellence Project of Zhejiang Province (No. 2007R10031).

\section{References}

[1] Arbarello, E.; DeConcini, C.; Kac, V.G.; Procesi, C., Moduli spaces of curves and representation theory. Comm. Math. Phys. 117 (1988), 1-36.

[2] Billig, Y., Representations of the twisted Heisenberg-Virasoro algebra at level zero, Canad. Math. Bull. 46 (2003), no. 4, 529-533.

[3] Chen, L., Differential operator Lie algebras on the ring of Laurent polynomials, Comm. Math. phys. 167 (1995), 431-469.

[4] Frenkel, I.B., Spinor representations of affine Lie algebras, Proc. Natl. Acad. Sci. USA, 77 (1980), 6303-6306.

[5] Feingold, A. J.; Frenkel, I.B., Classical affine Lie algebras, Adv. in Math. 56 (1985), 117-172.

[6] Fabbri, M.A.; Moody, R.V., Irreducible representations of Virasoro-toroidal Lie algebras, Comm. Math. Phys. 159 (1994), no. 1, 1-13.

[7] Fabbri, M.A.; Okoh, F., Representations of Heisenberg-Virasoro algebras and Virasorotoroidal algebras, Canad. J. Math. 51 (1999), no. 3, 523-545.

[8] Fu, J.Y.; Jiang, Q.F.; Su, Y.C., Classification of modules of the intermediate series over Ramond $N=2$ superconformal algebras, math.QA/0608508.

[9] Hu, N.; Liu, D., Decompositions of bosonic modules of Lie algebras $\mathcal{W}_{1+\infty}$ and $\mathcal{W}_{1+\infty}\left(g l_{N}\right)$, Chinese Math. Ann. 26(4) (Ser. B) (2005), 1-10. 
[10] Jiang, Q.F., Jiang, C.B., Representations of the twisted Heisenberg-Virasoro algebra and the full toroidal Lie Algebras, Algebra Colloquium 2007(14).

[11] Kong, X.L., Cheng, H.J., Bai, C.M, Classification of graded left-symmetric algebra structrue over Witt and virasoro algebras, Preprint. math QA/0706.4229.

[12] Kac, V.G.; van de Leur, J.W., On classification of superconformal algebras, Strings '88 (College Park, MD, 1988), 77-106.

[13] Kac, V.G.; Peterson, D.H., Spin and wedge representations of infinite-dimensional Lie algebras and groups, Proc. Natl. Acad. Sci. USA, 78 (1981), 3308-3312.

[14] Kac, V.G.; Radul, A., Quasifinite highest weight modules over the Lie algebra of differential operators on the circle, Comm. Math. Phys. 157 (1993), 429-457.

[15] Kaplansky, I.; Santharoubane, L. J., Harish-Chandra modules over the Virasoro algebra, Infinite-dimensional groups with applications (Berkeley, Calif., 1984), 217-231, Math. Sci. Res. Inst. Publ., 4, Springer, New York, 1985.

[16] Lu, R.; Zhao, K., Classification of irreducible weight modules over the twisted HeisenbergVirasoro algebra, math-ST/0510194.

[17] Osborn, J.M.; Zhao, K., Doubly Z-graded Lie algebras containing a Virasoro algebra, J. Alg., 219(1999), 266-298.

[18] Wang, W.Q., $W_{1+\infty}$ algebra, $W_{3}$ algebra, and Friedan-Martinec-Shenker bosonization, Comm. Math. Phys. 195 (1998), no. 1, 95-111. 